\title{
Preface: Gynecologic Urology
}

\author{
Eckhard Petri $\cdot$ Atsuo Kondo
}

Received: 7 December 2011 / Accepted: 12 December 2011/Published online: 5 January 2012

(C) Springer-Verlag 2012

The increasing interest in pelvic floor disorders, symptoms no longer being a taboo topic, awareness of the patients, and the industry recognizing a large market together with the urogenital aging of a demographically important population make gynecologic urology or urogynecology an important topic. These obvious facts are recognized around the world particularly at fellowship centers and international organizations, such as the International Continence Society (ICS) and the International Urogynecological Association (IUGA), and national societies, such as the American Urogynecologic Society (AUGS) and the Urodynamics Society (UDS) and many others. There are annual meetings (sometimes with $>2,000$ participants) and journals to support gynecologic urology.

The historical concept of three separate anatomical divisions of the pelvic floor-urological, gynecological, and colorectal-is herein rejected in favor of the philosophy that the pelvic floor is one functional unit, best managed by close collaboration between doctors and coworkers involved.

With this copy, the World Journal of Urology offers a wide spectrum of urogynecologic topics, covered by urologists, gynecologists, anatomists, physiotherapists, and other disciplines. Basic anatomy being discussed, conservative options including neuromodulation and the use of botulinum toxin are brought to your attention. The state of the art for anti-incontinence surgery as well as prolapse procedures are described and critically evaluated.

We hope this information is helpful in your clinical and practical work.

E. Petri $(\bowtie)$

University of Greifswald, Greifswald, Germany

e-mail: eckhard.petri@uni-greifswald.de

A. Kondo

Tsushima Rehabilitation Hospital, Tsushima, Japan 